\title{
Survival of Bacterial Biofilms within Neutrophil Extracellular Traps Promotes Nontypeable Haemophilus influenzae Persistence in the Chinchilla Model for Otitis Media
}

\author{
Wenzhou Hong Richard A. Juneau Bing Pang W. Edward Swords \\ Department of Microbiology and Immunology, Wake Forest University Health Sciences, Winston-Salem, N.C., USA
}

\author{
Key Words \\ Neutrophil extracellular trap $\cdot$ Survival $\cdot$ Biofilm $\cdot$ Otitis \\ media $\cdot$ Haemophilus influenzae
}

\begin{abstract}
Nontypeable Haemophilus influenzae (NTHi) is a leading cause of acute and chronic otitis media, which are a major public health problem worldwide. The persistence of NTHi during chronic and recurrent otitis media infections involves multicellular biofilm communities formed within the middle-ear chamber. Bacterial biofilms resist immune clearance and antibiotic therapy due in part to encasement within a polymeric matrix. In this study, the contribution of biofilms to bacterial persistence in vivo and composition of the NTHi biofilm matrix during experimental otitis media were investigated. The presence of biofilms within the chinchilla middle-ear chamber was significantly correlated with increased bacterial load in middle-ear effusions and tissue. Examination of thin sections revealed polymorphonuclear cells within a DNA lattice containing elastase and histones, which is consistent with the definition of neutrophil extracellular traps. Viable multicellular biofilm communities with biofilm phenotypes were found within the DNA lattice throughout the biofilm. Further, NTHi was resistant to both phagocytic and extracellular neutrophil killing in vitro by means of lipooligosaccharide moieties that promote biofilm formation. These data support the
\end{abstract}

conclusion that NTHi subverts neutrophil extracellular traps to persist in vivo. These data also indicate that a more inclusive definition for biofilms may be warranted.

Copyright $\odot 2009$ S. Karger AG, Basel

\section{Introduction}

Haemophilus influenzae exists as a component of the nasopharyngeal microbiota, and in most healthy people infection causes little or no adverse effect. In children, $H$. influenzae is among the most common causes of otitis media infections that are among the most common and costly pediatric diseases worldwide [1]. During chronic and recurrent otitis media, $H$. influenzae forms biofilm communities that are thought to promote bacterial persistence in vivo [2]. The most fundamental definition for a biofilm is a surface-adherent microbial community that resists immune or pharmaceutical clearance [3]. For $H$. influenzae, the biofilm production in vitro is widely shared among clinical isolates [4], and factors that promote biofilm formation include expression of a subset of lipooligosaccharide (LOS) glycoforms [5-7], type IV pili [8] and release of double-stranded DNA [9]. Many of

W.H. and R.A.J. contributed equally to this work.

\section{KARGER}

(C) 2009 S. Karger AG, Basel

Fax +4161306 1234

E-Mail karger@karger.ch

www.karger.com
Accessible online at:

www.karger.com/jin
Dr. W. Edward Swords

Department of Microbiology and Immunology

Wake Forest University Health Sciences, 5101A Gray Building

1000 Medical Center Boulevard, Winston-Salem, NC 27157 (USA)

Tel. +1 336713 5049, Fax +1 336716 9928, E-Mail wswords@wfubmc.edu 
these studies have included infection studies in the chinchilla or other infection models to validate the relevant genotype in the contexts of persistence and virulence $[5$, $6,8-11]$.

The formation of a polymeric matrix material that encases a bacterial community is a widely shared property of biofilms that may promote resistance to clearance by host immunity and antibiotics [12]. In this study, we examined biofilms recovered from the chinchilla middleear chamber to better define the matrix of the $H$. influenzae biofilm. As previously reported, biofilms contained a large proportion of fibrous DNA material [9]. Host neutrophils were also observed throughout the biofilm, and eukaryotic histones and elastase were observed within the DNA matrix. The composition and structure of these communities was thus consistent with neutrophil extracellular traps (NETs). Communities of viable $H$. influen$z a e$ bacteria were also observed within the NET structure. Using an in vitro NET bactericidal assay, $H$. influenzae was shown to survive within NETs in a manner that was dependent upon the composition of LOS glycolipids on the bacterial surface. LOS mutants with impaired survival in NETs included those with previously established effects on biofilm formation and integrity. Therefore, we conclude that $H$. influenzae subverts the process of NET formation to form persistent biofilms in vivo.

\section{Materials and Methods}

\section{Bacterial Strains}

H. influenzae strains 2019 and 86-028NP, and their derivatives, were used in this study. The relevant genotypes and phenotypes of all bacterial strains are provided in table 1 . All bacterial strains were cultured on brain-heart infusion agar (Difco) supplemented with hemin and NAD, as described previously $[13,14]$.

\section{Infections}

Healthy adult chinchillas (400-800 g) were obtained from Rauscher's Chinchilla Ranch and were housed for 7-10 days after shipping to minimize the impact of shipping-related stress, after which transbullar infections were carried out essentially as described previously $[6,11]$. $H$. influenzae bacteria were harvested from supplemented brain-heart infusion plates and suspended in sterile phosphate-buffered saline to an $\mathrm{OD}_{600}$ of 0.15 (approx. $10^{8}$ bacteria/ml). Chinchillas were anesthetized with isofluorane gas and infected via transbullar injection with $0.1 \mathrm{ml}$ of suspension containing approximately 1,000 CFU/ear. Infectious doses were confirmed by plate count of inocula and varied less than $15 \%$ between infection groups. Establishment and progression of otitis media was assessed by otoscopic examination at 48-hour intervals. Groups of animals were euthanized at 7 and 14 days following infection, after which the superior bullae were excised, exposing the middle-ear chamber. Macroscopically visible biofilms were ex-
Table 1. List of NTHi strains used in this study

\begin{tabular}{lll}
\hline Strain & Relevant phenotype & Ref. \\
\hline H. influenzae 86-028NP & otitis isolate & 38 \\
H. influenzae 2019 & bronchitis isolate & 39 \\
H. influenzae 2019 htrB & underacylated lipid A & 40,41 \\
H. influenzae 2019 rfaD & core assembly defect & 42 \\
H. influenzae 2019 rfaF & core assembly defect & 42 \\
\hline
\end{tabular}

cised, along with the underlying mucosal surface, and processed as described below. All animal infections were carried out according to protocols approved by the Wake Forest University Health Sciences (WFUHS) Animal Care and Use Committee.

\section{Microscopy}

Biofilms and middle-ear tissue were excised from the middleear chambers of chinchillas immediately following euthanasia as described previously $[6,11]$. For viability staining, portions of the biofilm were cut into small pieces $(2-3 \mathrm{~mm})$, and incubated with $0.5 \mathrm{ml}$ of PBS containing a mixture of SYTO 9 and propidium iodide for $15 \mathrm{~min}$, and then washed 3 times with PBS buffer. Samples were visualized using a Zeiss LSM510 confocal laser scanning microscope with an argon laser and 488 and $543 \mathrm{~nm}$ filters, using Zeiss LSM Image Browser software. Additional portions of the biofilms were processed for immunofluorescence and electron microscopy. For these analyses, samples were fixed briefly in $1 \%$ paraformaldehyde/PBS and rinsed in PBS ( 2 washes, $5 \mathrm{~min} /$ wash). For cryosection, biofilm was embedded in OCT resin (Sakura Finetek). After storage overnight at $-20^{\circ} \mathrm{C}$, the samples were cut into sections $(5 \mu \mathrm{m})$ using a cryotome, and placed on to adhesive slides. Blocks and sections were stored at $-20^{\circ} \mathrm{C}$ prior to further analyses. For immunofluorescent staining, the slides were brought to room temperature, and then fixed briefly with $2 \%$ paraformaldehyde-PBS prior to antibody and/or lectin staining as indicated in the text. Individual sections were stained with rabbit antisera directed against $H$. influenzae [11], anti-histone (panreactive monoclonal antibody MAB3422; Chemicon) or anti-elastase (monoclonal antibody NP57; Thermo Scientific), followed with appropriate fluorescent antibody conjugates as indicated in the text. All fluorescent antibody conjugates were obtained from Jackson Laboratories. For lectin staining, sections were stained with the sialic acid-specific Limax flavus lectin conjugated to rhodamine (EY Laboratories), as we have described previously [11, 15]. Immunofluorescence microscopy was performed using a Zeiss LSM 510 confocal laser-scanning microscope. Quantification of fluorescence was performed by pixel counts using Photoshop software as indicated in the figure legends.

For histological examination, sections were stained with toluidine blue according to standard methodology, and viewed on a Nikon Eclipse microscope. For scanning electron microscopy, portions of the biofilms were fixed for $60 \mathrm{~min}$ with $2.5 \%$ glutaraldehyde-PBS and then rinsed twice (10 min/wash) in PBS prior to dehydration in a graded ethanol series. The samples were then subjected to critical point drying, mounted onto stubs, and sputter coated with palladium prior to viewing with a Philips SEM515 scanning electron microscope. 
Fig. 1. Biofilms formed by $H$. influenzae 86-020NP promote bacterial persistence in the chinchilla middle-ear chamber. Animals were infected via transbullar injection, and groups were euthanized at 7 and 14 days after infection. a Biofilm formation in the chinchilla middle-ear chamber. Photographic images in the top row show representative middle-ear chambers of mock-infected animal (left) as well as infected animals with macroscopically visible biofilms 7 days (center) and 14 days (right) after infection. Scanning electron micrographs (SEM) in the lower row show epithelial surface of the middle-ear chamber of a mock-infected animal (left) compared to infected animals 7 days (center) and 14 days (right) after infection. Note the visible biomass in the infected animals that contains embedded host cells in the 14-day image. b Bacterial counts obtained from middle-ear effusion fluids of animals with and without visible biofilm. Bars depict mean CFU counts from infected animals 7 days (left) or 14 days (right) after infection. Sample size: biofilm group, $\mathrm{n}=31$; no biofilm group, $\mathrm{n}=9$. Error bars show standard deviation. Statistical significance was assessed by Mann-Whitney nonparametric analysis and is denoted by an asterisk.

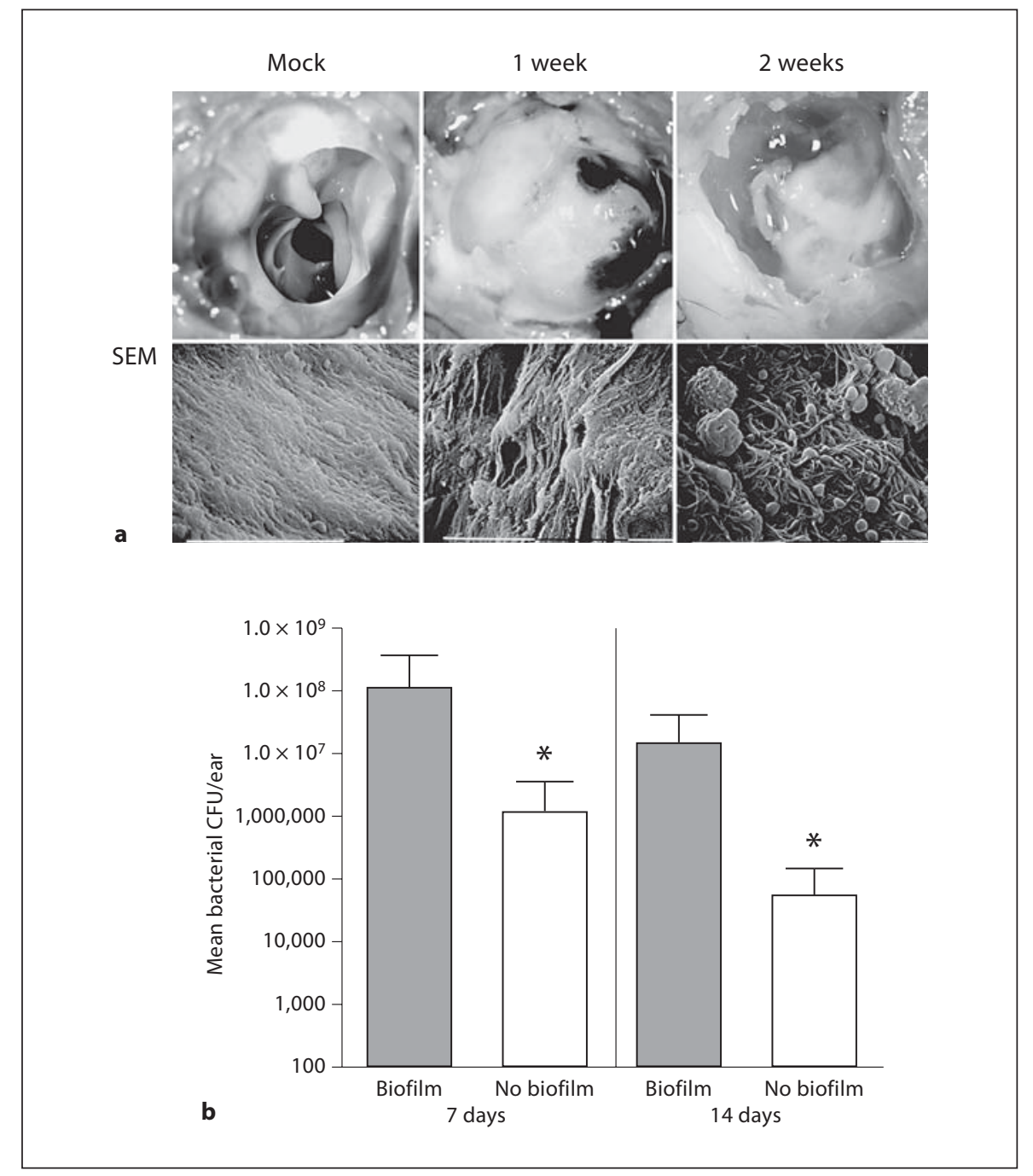

\section{Neutrophil Killing Assays}

Neutrophils were isolated from peripheral blood obtained from healthy donors according to standard method using Isolymph density-gradient centrifugation. All procedures for blood draws were reviewed and approved by the WFUHS Institutional Review Board. Measurements of bactericidal activity of neutrophils via phagocytic and NET routes of killing were performed essentially as described previously [16]. Neutrophils (approx. 106/well) were treated with $25 \mathrm{nM}$ phorbol myristate acetate (PMA; Sigma) for 10 min to elicit NET formation, which was confirmed in control experiments by Live/Dead ${ }^{\mathrm{TM}}$ stain, as described above, and fluorescence microscopy. RPMI medium (with or without $20 \mu \mathrm{M}$ cytochalasin D) was added to the neutrophils and incubated for $15 \mathrm{~min}$. Bacteria were added $\left(10^{4} \mathrm{CFU}\right.$, approx. multiplicity of infection $=$ 0.01 ) and incubated for $30 \mathrm{~min}$, after which the wells were scraped and plate counts performed. Bactericidal activity was expressed as a percentage of counts obtained from control wells in medium with no neutrophils. NET-mediated killing was obtained from similar comparisons in the presence of cytochalasin, with or without neu- trophils. Complete inhibition of $H$. influenzae phagocytosis by neutrophils in the presence of $20 \mu \mathrm{m}$ cytochalasin D was confirmed in control experiments (data not shown).

\section{Statistical Analysis}

Data were analyzed by nonparametric t test or analysis of variance with a post hoc test of significance, as indicated in the text.

\section{Results}

\section{Host Cells Are Visible within Nontypeable}

$\mathrm{H}$. influenzae Biofilms in vivo

Nontypeable $H$. influenzae (NTHi) 86-028NP forms opaque communities within the chinchilla middle-ear chamber (fig. 1a) that have been termed biofilms [6, 8-11, 17, 18]. The basis for this designation, as elucidated 


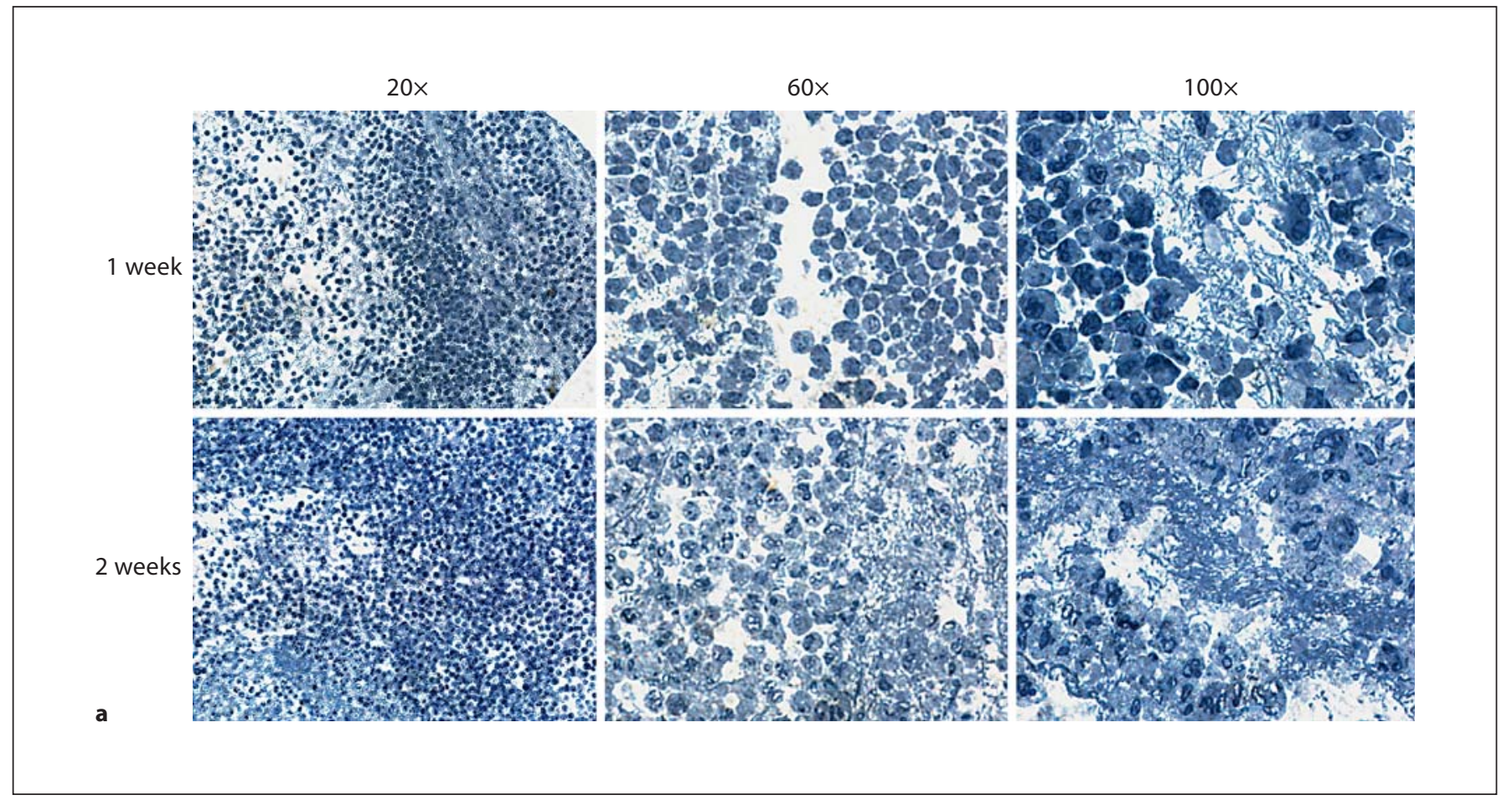

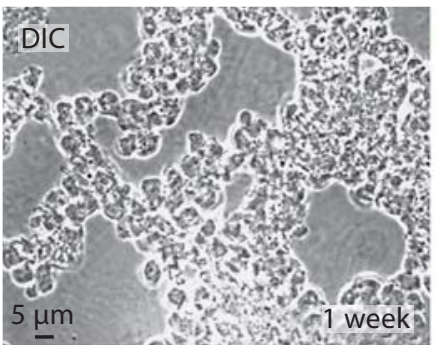
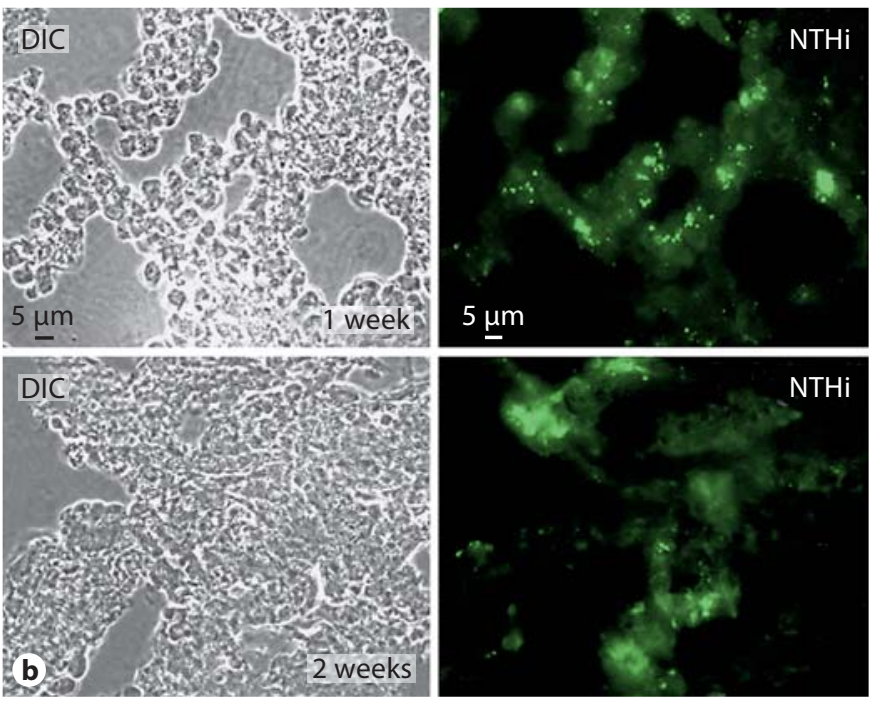

Fig. 2. Visualization of $H$. influenzae communities within cryosections of biofilms recovered from the chinchilla middle ear. Biofilms recovered from infected animals were sectioned and analyzed microscopically. a Histopathological analysis reveals neutrophils within the biofilm. Sections of biofilm from animals at 1 and 2 weeks after infection were stained with toluidine blue, and revealed many polymorphonuclear cells embedded within the biofilm structure. b Immunofluorescent staining to visualize $H$. influenzae bacteria within biofilm. Cryosections $(5 \mu \mathrm{m})$ were stained with polyclonal rabbit antisera against $H$. influenzae 86 -

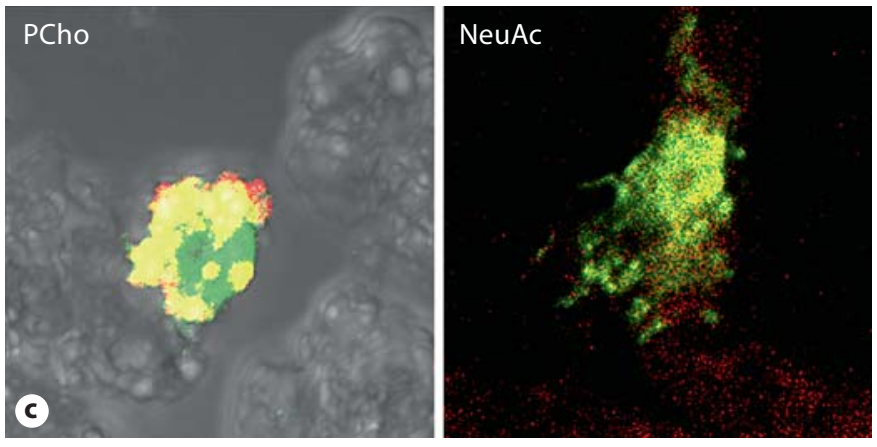

$028 \mathrm{NP}$ and anti-rabbit Alexa488 conjugate and visualized by differential interference contrast (DIC)/Nomarski and fluorescence microscopy. $H$. influenzae communities are visible in green in fluorescent panel at both the 1- and 2-week postinfection time points. c Bacterial variants within communities within the biofilm. Cryosections were stained with polyclonal antiserum as above coupled with monoclonal antibody hyaluronan synthases specific for phosphorylcholine (PCho, left image) and secondary antibody Texas Red conjugate, or lectin LFA/rhodamine conjugate for sialic acid (NeuAc, right image). 


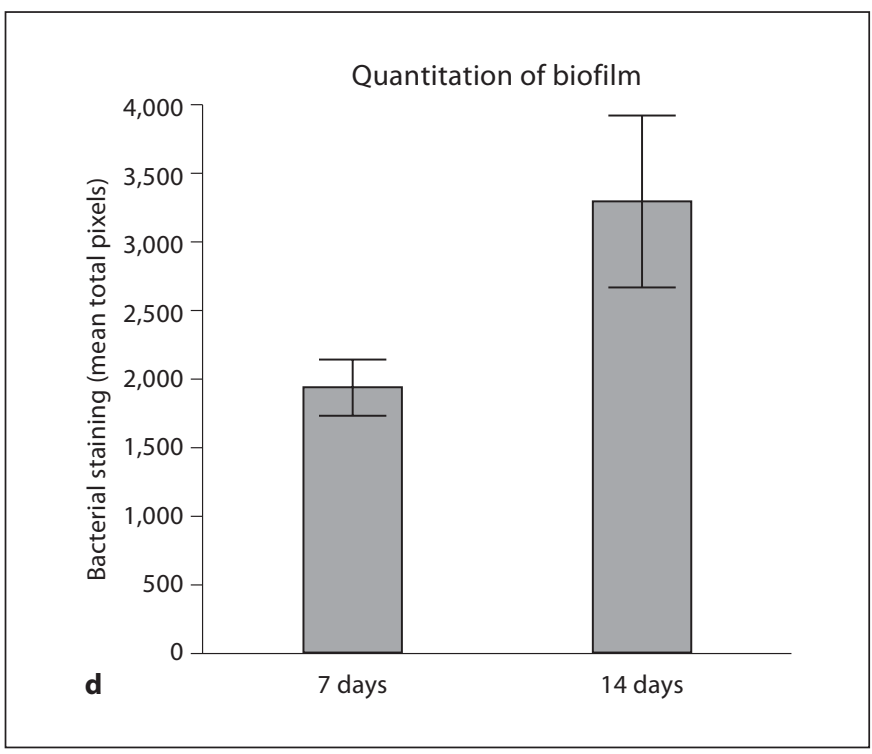

Fig. 2. d Quantitation of biofilm within cryosections. Graph depicts mean fluorescent pixel counts from 5 different fields of view of 20 different sections. Pixels with similar intensity (within 20\%) were highlighted. Total pixel counts for images were comparable (within 15\%). Error bars depict standard deviation.

through a series of studies from several different laboratories, was that the bacterial community was found associated with host surfaces and was encased in an electron-dense matrix material $[6,8-11,17]$. Analysis of this community by scanning electron microscopy reveals the presence of an electron-dense matrix component and host cells associated with and integrated within the biofilm structure (fig. 1a). In order to address the contribution of the biofilm to bacterial persistence, we compared bacterial counts obtained from chinchilla middle-ear chambers with macroscopically visible biofilms with counts from ears lacking biofilms (fig. 1b). The results clearly showed significantly higher bacterial counts in ears containing biofilms than in those lacking visible biofilm.

Biofilms recovered from infected chinchillas were sectioned and examined by histopathological and immunohistochemical stain (fig. 2a). In toluidine blue-stained sections at 1 and 2 weeks after infection, many immune cells (predominantly polymorphonuclear cells such as neutrophils or mast cells) were observed within the biofilm structure lining open spaces (water channels) within the biofilm structure (fig. 2a). Notably, there were few polymorphonuclear cells visible within the condensed portions of the biofilm. Fluorescent antibody staining us- ing antisera specific for $H$. influenzae revealed bacterial communities within a mass that was not reactive with the antisera, thus indicating that it was not composed of bacteria or bacterial-derived material (fig. 2b). Staining of the sections with monoclonal antibody (hyaluronan synthases recognizing phosphorylcholine, PCho) or lectin (Limax flavus lectin recognizing sialic acid, NeuAc) specific for bacterial surface moieties associated with biofilms shows that the bacterial aggregates contain NTHi variants found within biofilm communities (fig. 2c). Notably, our previous work has shown that both sialylated and PCho+ variants are both found within biofilm communities in vitro and in vivo, and promote formation of biofilms $[5,6,11]$. Thus, the current results provide support for the designation of these communities as biofilms. The bacterial aggregates grew larger during the course of the infection, increasing by approximately 2 -fold from 1 to 2 weeks after infection, as assessed by fluorescent pixel counts (fig. 2d). These results are consistent with the biofilm hypothesis for bacterial persistence during chronic and recurrent otitis media.

\section{Viable Bacteria and Host Cells Are Visible within a}

DNA Matrix in NTHi Biofilms

Portions of $H$. influenzae biofilms recovered from the chinchilla infections were examined by Live/Dead staining (Molecular Probes). For biofilms obtained at 1 and 2 weeks after infection, viable host and bacterial cells are visible within a fibrous matrix that stains with propidium iodide, and is thus consistent with DNA (fig. 3). These data were consistent with the DNA matrix material observed previously for NTHi biofilm communities in vitro and in vivo [9]. However, we recognized that these images were also consistent with the current definition of NET structures that form as part of a secondary, extracellular means for invading neutrophils and other phagocytes (most notably mast cells) to trap and kill microbes within a fibrous DNA matrix $[19,20]$. Defining characteristics of NET structures include the presence of host histones and elastase within the NET and attached to the DNA fibers. Thus, we stained cryosections of the in vivo biofilms with antibodies recognizing both histones and elastase (fig. 4). These images clearly show that elastase and histone are present within the biofilm in close proximity with $H$. influenzae bacteria. However, in many regions within the stained sections, the histone and elastase staining was not observed throughout the bacterial aggregates, and thus the NET bactericidal factors did not appear to permeate completely into the bacterial communities. Thus, the surface-attached material containing $H$. 
$\mathrm{PI}$
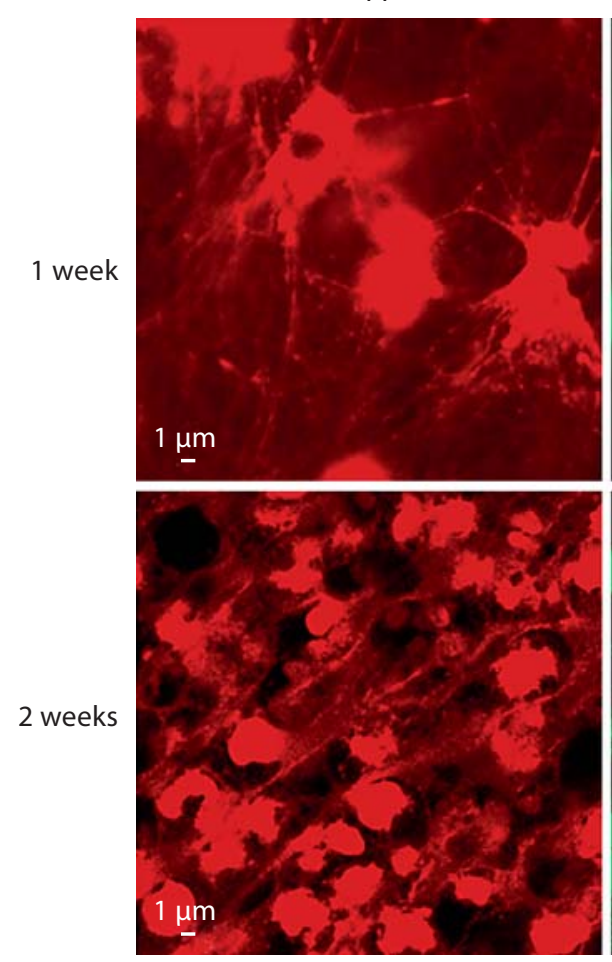

Syto-9 green
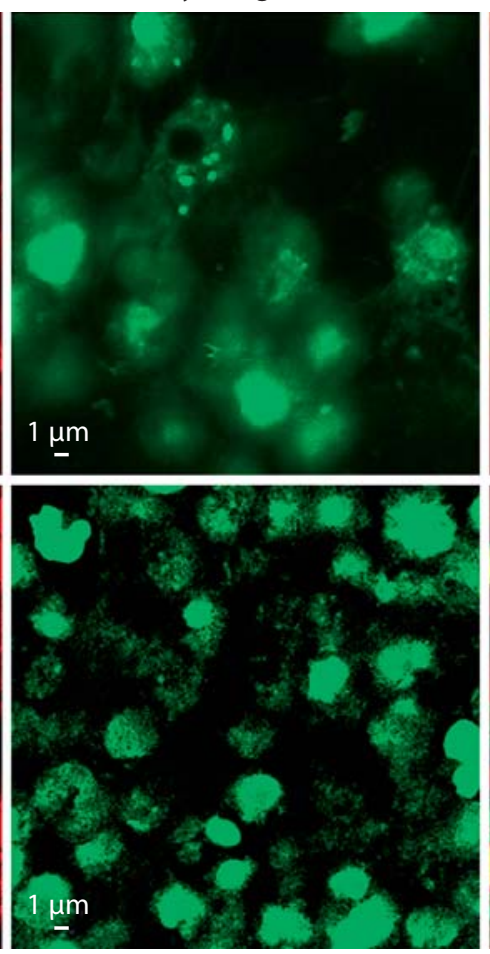
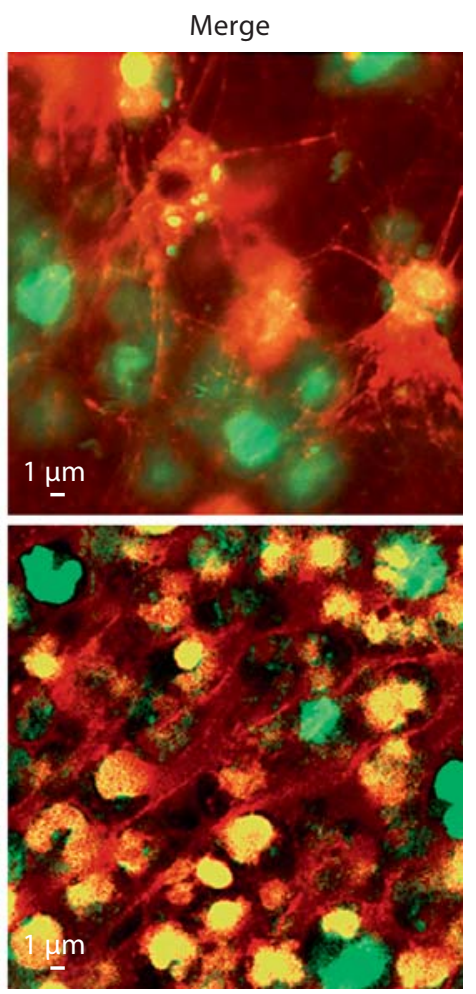

Fig. 3. Viability staining reveals host cells and bacteria within a fibrous DNA lattice. Unfixed portions of biofilms were stained with Live/Dead BacLight ${ }^{\mathrm{TM}}$ (Molecular Probes) and visualized by confocal laser-scanning microscopy. With this reagent, red staining (propidium iodide, PI) indicates nonviable cells or extracellular DNA, whereas green staining (Syto-9) indicates viable cells.
Images depicted are merged from vertical $\mathrm{Z}$ sections $(0.2 \mu \mathrm{m} /$ slice, 5 sections per image). Biofilms were recovered 1 week (top row) or 2 weeks (bottom row) after infection. Viable bacteria and both viable and nonviable host cells were observed within fibrous DNA net in samples from both time points. influenzae biofilms includes a significant polymorphonuclear cell extracellular trap component that does not appear to mediate clearance.

\section{LOS on the H. influenzae Bacterial Surface Provide Resistance to NET Killing}

Because our prior data have shown both $H$. influenzae bacteria with biofilm properties present within surfaceadherent communities in vivo, we hypothesized that the factors involved in formation/stability of biofilms might promote survival of bacterial communities within a NET structure. Thus, we used an in vitro assay for NET bactericidal activity to ask whether NETs effectively kill $H$. influenzae bacteria. Neutrophils were treated with PMA (25 nM) as described previously [19], and formation of
NETs was confirmed by visualization of the DNA lattice in control wells following propidium iodide staining. Bacteria were added at a low multiplicity of infection (0.1) and incubated for $30 \mathrm{~min}$, followed by plate count. As shown in figure 5, while $H$. influenzae 2019 was killed (albeit inefficiently) by PMA-treated neutrophils, inhibition of phagocytosis by pretreatment with cytochalasin completely abolishes this killing. Thus, wild-type $H$. influenzae bacteria are resistant to killing within NET structures. In order to test the contribution of the LOS on the bacterial surface to $H$. influenzae survival in NETs, we compared survival/killing of a series of mutants with altered LOS composition and structure. As expected, $H$. influenzae 2019 htrB, $r f a D$ and $r f a F$ mutants with core/ lipid A assembly defects were more readily susceptible to 
Fig. 4. Immunofluorescence microscopy reveals eukaryotic histones and elastase within the biofilm structure. Cryosections from biofilms obtained 1 or 2 weeks after infection (as indicated in labels on left margin) were stained with rabbit antisera against $H$. influenzae coupled with secondary antibody/Texas Red conjugate as above, coupled with monoclonal antibody specific for elastase and secondary antibody/Alexa488 conjugate (top) or deiminated histone/Alexa488 conjugate (bottom). The stained sections were visualized by confocal laser-scanning microscopy; the images depicted are merged from vertical $\mathrm{Z}$ sections $(0.2 \mu \mathrm{m} /$ slice, 10 sections per image).
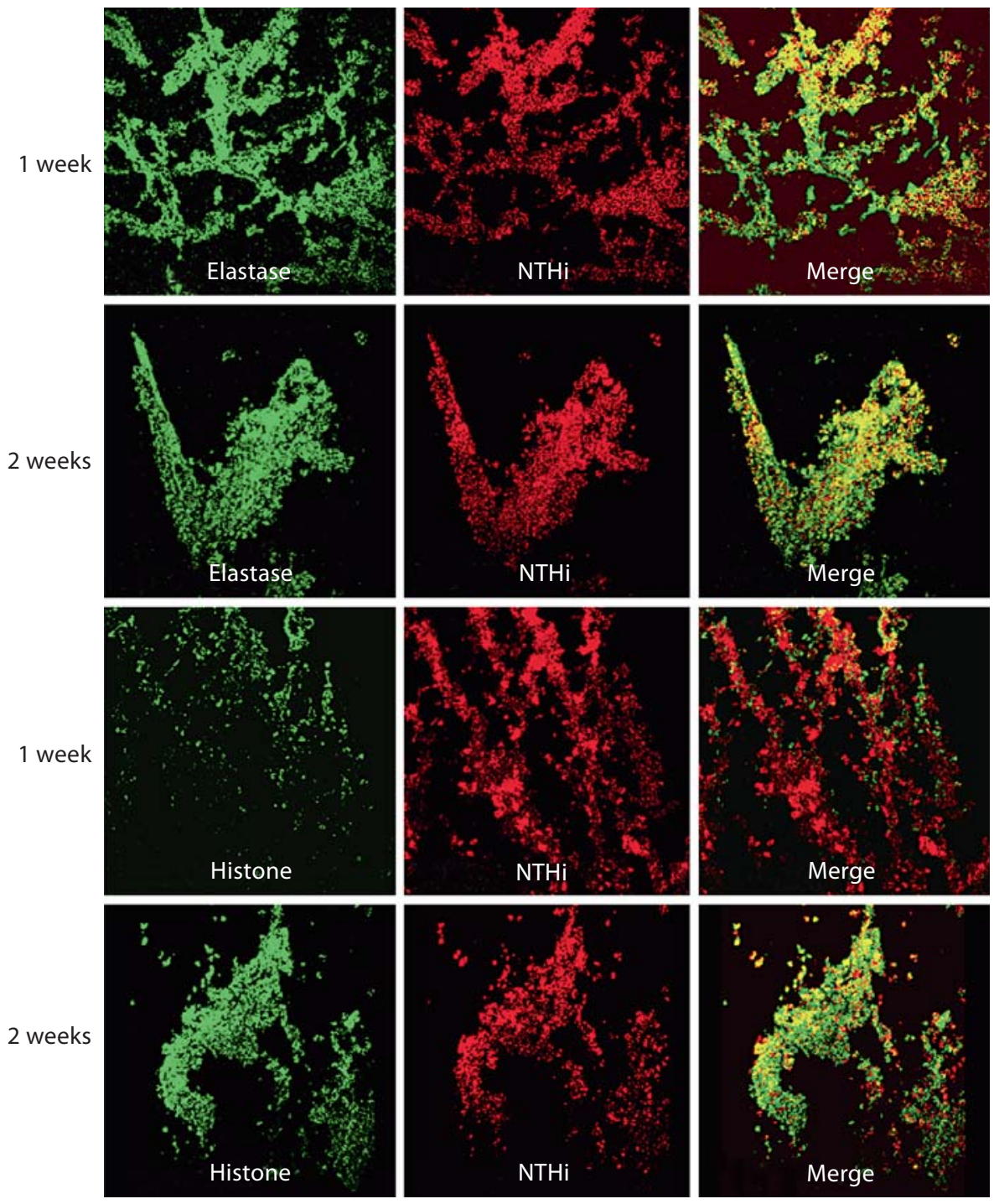

NET killing, with $25-45 \%$ of the bacterial populations being killed even when phagocytosis was inhibited by cytochalasin. Notably, previous work has shown that the NTHi 2019 htrB and $r f a D$ mutants are attenuated in the chinchilla model for otitis media [21]. Because prior work has shown that the oligosaccharide portion of the LOS is important in biofilm formation in vitro and in vivo, we tested NET survival of $H$. influenzae mutants with alterations in this part of the LOS molecule. $H$. influenzae 2019 pgmB has a biosynthetic defect that results in a drastically truncated LOS oligosaccharide region [13] and also has a biofilm phenotype [10]. H. influenzae 2019 siaB is an asialylated mutant that is deficient in biofilm formation and persistence in vivo $[5,7,22]$. Using the NET bactericidal assay, both of these mutants were shown to be more readily killed within NETs than the parental strain by both phagocytic and extracellular routes (fig. 5). These results are consistent with our hypothesis that the biofilm phenotype confers resistance to clearance by NETs.

\section{Discussion}

Biofilms are defined as surface-associated multicellular communities that promote bacterial persistence [23, 24]. In many cases, bacterial species produce a polymeric matrix material that may contribute to the persistence phenotype, possibly by promoting resistance to host immune effectors and antimicrobials [3]. The composition of biofilm matrix is variable and can include polysaccha- 


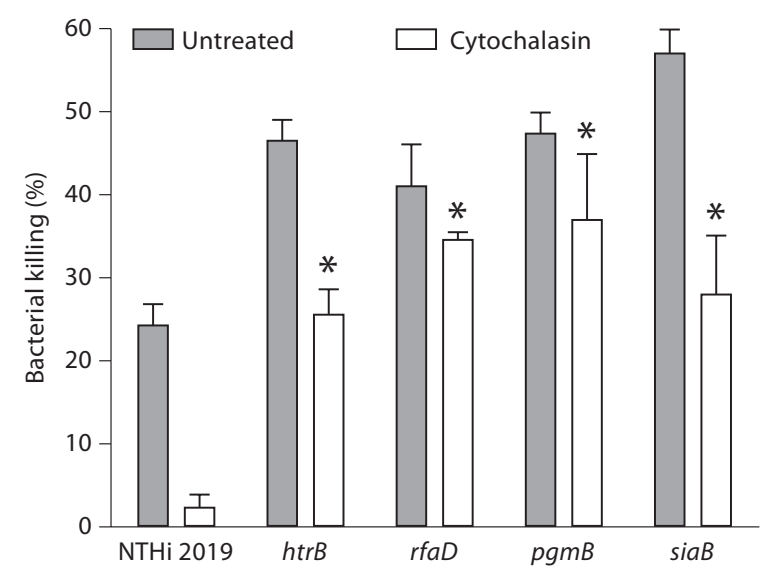

Fig. 5. $H$. influenzae resists killing within preformed NETs. NET formation was elicited from primary human neutrophils by stimulation with PMA (see Materials and Methods), after which NTHi bacteria were added. After $30 \mathrm{~min}$, the number of viable bacteria within the well was determined by plate count and normalized relative to mock-treated wells to which no neutrophils were added. Filled columns show means of total neutrophil killing (absence of cytochalasin), whereas open columns show means of extracellular killing (wells treated with cytochalasin). Error bars show standard deviation, while asterisks denote mean values that were significantly different from the parental NTHi 2019 strain as determined by analysis of variance with a post hoc test of significance. NET killing was defined as the percentage of decrease in total bacteria in the presence of neutrophils and cytochalasin D.

ride, nucleic acid and/or proteins [25]. For H. influenzae, several studies, including those from our group, have shown that biofilms cultured in vitro and in vivo are encased in an electron-dense material that has been referred to as matrix [5-11, 15]. Sialylated LOS, and possibly a non-LOS polysaccharide, are essential to $H$. influenzae biofilm formation in vitro and in vivo $[5,7,10,26,27]$. Data supporting a role for sialylated moieties as the matrix of $H$. influenzae biofilms include staining of material between bacteria within biofilms with sialyl-reactive lectins [10]. However, $H$. influenzae biofilms cultured in continuous-flow systems in vitro and recovered from the chinchilla middle-ear chamber also contain extracellular DNA [9], as has been observed for biofilms formed by other species [28-31]. The data in this study support the conclusion that a substantial portion of the nucleic acid in $H$. influenzae biofilms in vivo is derived from host cells such as neutrophils or mast cells. NETs are formed after exposure to a variety of microbial stimuli and can medi- ate bacterial killing primarily via neutrophilic components such as elastase and histones that are attached to a web of DNA fibers [19]. Additional work shows that mast cells can also form similar structures that have bactericidal activity, too [20]. Of note, mast cells have been demonstrated to be involved in the inflammatory response during otitis media $[32,33]$. The data presented in this study clearly show both elastase and histones attached to a fibrous nucleic acid network within $H$. influenzae biofilms recovered from the chinchilla middle-ear cavity. Thus, one might postulate that the biofilm communities observed in this animal model are composed of bacteria that are ultimately cleared. However, our data clearly do not support this conclusion, as the presence of biofilms was associated with higher bacterial numbers within middle-ear fluids and tissues. Moreover, viable bacterial communities were observed interspersed throughout the biofilm structure by viability staining. These data are in agreement with our prior work using colony counts $[5,6$, 11]. It is also important to note that these bacterial communities exhibited 2 of the hallmarks of $H$. influenzae biofilms cultured using in vitro biofilm models: the presence of variants expressing NeuAc as well as PCho within the biofilm and of extracellular DNA within the biofilm structure $[5-7,10,11]$. We have also clearly demonstrated that $H$. influenzae bacteria survive within a preformed NET structure in vitro, and this survival was dependent on the expression of sialylated LOS glycoforms on the bacterial surface. It is noteworthy that the $\mathrm{H}$. influenzae mutants used in these studies were previously shown to be attenuated in infection models for otitis media [10, 21]. Work from our group and others has demonstrated that specific LOS glycoforms are required for the formation and maturation of biofilms in vitro $[5,6]$ as well as that these LOS forms promote bacterial persistence in vivo $[5,6,11,15]$. The present data thus support the conclusion that the $H$. influenzae bacteria persisting within NET structures are biofilms. Based on our data we propose that in at least some settings one contribution of the biofilm mode of NTHi growth may be to promote resistance to killing by bactericidal factors within NET structures. It is also plausible that these communities provide resistance to phagocytic killing by neutrophils. On that point, the data from in vitro bactericidal assays clearly show that $H$. influenzae bacteria are not readily killed by the phagocytic route (that is, in the absence of cytochalasin). These findings are consistent with previous reports showing that NTHi strains are highly resistant to phagocytic killing by neutrophils and macrophages $[34,35]$. 
Most in the field include presence of a matrix of bacterial origin as a defining characteristic for bacterial biofilms. We view this as a somewhat arbitrary and limiting definition, as within the context of bacterial pathogenesis, the true hallmark of biofilms is persistence in the face of bactericidal factors. In the case of NTHi, the current data show that formation of NET structures by incoming neutrophils significantly contributes to biofilm formation. This is consistent with at least one prior study with Pseudomonas aeruginosa, in which addition of neutrophils enhanced the formation and density of biofilms [36]. Given the failure of NETs to clear NTHi biofilms, it seems clear that the primary contribution of these structures during chronic otitis media is to facilitate establishment of a stable biofilm community. Continual neutrophilic influx and bacterial persistence are commonly associated with most clinical presentations of chronic opportunistic airway infections. Notably, we have also observed significant numbers of neutrophils within pneu- mococcal biofilms in the chinchilla model for otitis media [37]; it is thus plausible that, for bacteria with the ability to resist NET-mediated clearance, the persistence of bacterial biofilm communities within NET structures may be a common and heretofore unappreciated means for bacterial resistance to clearance.

\section{Acknowledgements}

The authors thank Drs. Sean Reid, Jason Grayson, Marlena Westcott and Dan Wozniak for helpful conversations and critiques of the manuscript. Ken Grant, Bilinda Dawson and other members of the WFUHS microscopy core facility provided expert assistance in electron microscopy. Gayle Foster provided techni$\mathrm{cal}$ assistance in the chinchilla studies. Staff of the WFUHS Animal Research Program provided invaluable assistance with infection studies. This work was funded by grants from NIH/NIDCHD (DC007444), NIH/NIAID (AI054425) and the Whitaker Infectious Disease fund.

\section{References}

1 Klein JO: The burden of otitis media. Vaccine 2000;19:S2-S8.

- Bakaletz LO: Bacterial biofilms in otitis media: evidence and relevance. Pediatr Infect Dis J 2007;26:S17-S19.

- 3 Fux CA, Costerton JW, Stewart PS, Stoodley P: Survival strategies of infectious biofilms. Trends Microbiol 2005;13:34-40.

4 Murphy TF, Kirkham C: Biofilm formation by nontypeable Haemophilus influenzae: strain variability, outer membrane antigen expression and role of pili. BMC Microbiol 2002;2:7.

5 Swords WE, Moore ML, Godzicki L, Bukofzer G, Mitten MJ, VonCannon J: Sialylation of lipooligosaccharides promotes biofilm formation by nontypeable Haemophilus influenzae. Infect Immun 2004;72:106-113.

-6 Hong W, Pang B, West-Barnette S, Swords WE: Phosphorylcholine expression by nontypeable Haemophilus influenzae correlates with maturation of biofilm communities in vitro and in vivo. J Bacteriol 2007;189:83008307.

7 Greiner L, Watanabe H, Phillips NJ, Shao J, Morgan A, Zaleski A, Gibson BW, Apicella MA: Nontypeable Haemophilus influenzae strain 2019 produces a biofilm containing $\mathrm{N}$ acetylneuraminic acid that may mimic sialylated O-linked glycans. Infect Immun 2004;72:4249-4260.
8 Jurcisek JA, Bookwalter J, Baker B, Fernandez S, Novotny LA, Munson RS Jr, Bakaletz LO: The PilA protein of nontypeable Haemophilus influenzae plays a role in biofilm formation, adherence to epithelial cells and colonization of the mammalian upper respiratory tract. Mol Microbiol 2007;65:12881299.

9 Jurcisek JA, Bakaletz LO: Biofilms formed by nontypeable Haemophilus influenzae in vivo contain both double-stranded DNA and type IV pilin protein. J Bacteriol 2007;189: 3868-3875.

10 Jurcisek JA, Greiner L, Watanabe H, Zaleski A, Apicella MA, Bakaletz LO: Role of sialic acid and complex carbohydrate biosynthesis in biofilm formation by nontypeable Haemophilus influenzae in the chinchilla middle ear. Infect Immun 2005;73:3210-3218.

-11 Hong W, Mason K, Jurcisek JA, Novotny LA, Bakaletz LO, Swords WE: Phosphorylcholine decreases early inflammation and promotes the establishment of stable biofilm communities of nontypeable Haemophilus influenzae strain 86-028NP in a chinchilla model of otitis media. Infect Immun 2007; 75:958-965.

12 Donlan RM: Biofilms: microbial life on surfaces. Emerg Infect Dis 2002;8:881-890.

13 Swords WE, Buscher B, Ver Steeg K, Nichols W, Preston A, Weiser JN, Gibson B, Apicella MA: Nontypeable Haemophilus influenzae adhere to and invade human bronchial epithelial cells by an interaction of lipooligosaccharide with the PAF receptor. Mol Microbiol 2000;37:13-27.
14 Swords WE, Ketterer MR, Shao J, Campbell CA, Weiser JN, Apicella MA: Binding of the nontypeable Haemophilus influenzae lipooligosaccharide to the PAF receptor initiates host cell signaling. Cell Microbiol 2001;8: 525-536.

15 West-Barnette S, Rockel A, Swords WE: Biofilm growth increases phosphorylcholine content and decreases potency of nontypeable Haemophilus influenzae endotoxins. Infect Immun 2006;74:1828-1836.

16 Wartha F, Beiter K, Albiger B, Fernebro J, Zychlinsky A, Normark S, Henriques-Normark B: Capsule and D-alanylated lipoteichoic acids protect Streptococcus pneumoniae against neutrophil extracellular traps. Cell Microbiol 2007;9:1162-1171.

17 Post JC: Direct evidence of bacterial biofilms in otitis media. Laryngoscope 2001;111: 2083-2094

18 Ehrlich GD, Veeh R, Wang X, Costerton JW, Hayes JD, Hu FZ, Daigle BJ, Ehrlich MD, Post JC: Mucosal biofilm formation on middle-ear mucosa in the chinchilla model of otitis media. JAMA 2002;287:1710-1715.

19 Brinkmann V, Reichard U, Goosmann C, Fauler B, Uhlemann Y, Weiss DS, Weinrauch Y, Zychlinsky A: Neutrophil extracellular traps kill bacteria. Science 2004;303:15321535.

20 von Kockritz-Blickwede M, Goldmann O, Thulin P, Heinemann K, Norrby-Teglund A, Rohde M, Medina E: Phagocytosis-independent antimicrobial activity of mast cells by means of extracellular trap formation. Blood 2008;111:3070-3080. 
-21 DeMaria TF, Apicella MA, Nichols WA, Leake ER: Evaluation of the virulence of nontypeable Haemophilus influenzae lipopolysaccharide $h t r B$ and $r f a D$ mutants in the chinchilla model of otitis media. Infect Immun 1997;65:4431-4435.

-22 Jones PA, Samuels NA, Phillips NJ, Munson RS, Bozue JA, Arseneau JA, Nichols WA, Zaleski A, Gibson BW, Apicella MA: Haemophilus influenzae type B strain A2 has multiple sialyltransferases involved in lipooligosaccharide sialylation. J Biol Chem 2002;277: 14598-14611.

23 Costerton JW, Stewart PS, Greenberg EP: Bacterial biofilms: a common cause of persistent infections. Science 1999;284:13181322.

24 Costerton JW, Lewandowski Z, Caldwell DE, Korber DR, Lappin-Scott HM: Microbial biofilms. Annu Rev Microbiol 1995;49: 711-745.

$>25$ Flemming HC, Neu TR, Wozniak DJ: The EPS matrix: the 'house of biofilm cells'. J Bacteriol 2007; 189:7945-7947.

26 Pang B, Hong W, West-Barnette SL, Kock ND, Swords WE: Diminished ICAM-1 expression and impaired pulmonary clearance of nontypeable Haemophilus influenzae in a mouse model for COPD/emphysema. Infect Immun 2008;76:4959-4967.

-27 Pang B, Winn D, Johnson R, Hong W, WestBarnette S, Kock N, Swords WE: Lipooligosaccharides containing phosphorylcholine delay pulmonary clearance of nontypeable Haemophilus influenzae. Infect Immun 2008;76:2037-2043.

-28 Steinberger RE, Holden PA: Extracellular DNA in single- and multiple-species unsaturated biofilms. Appl Environ Microbiol 2005;71:5404-5410.
29 Allesen-Holm M, Barken KB, Yang L, Klausen M, Webb JS, Kjelleberg S, Molin S, Givskov M, Tolker-Nielsen T: A characterization of DNA release in Pseudomonas aeruginosa cultures and biofilms. Mol Microbiol 2006; 59:1114-1128.

30 Rice KC, Bayles KW: Molecular control of bacterial death and lysis. Microbiol Mol Biol Rev 2008;72:85-109.

31 Rice KC, Mann EE, Endres JL, Weiss EC, Cassat JE, Smeltzer MS, Bayles KW: The cidA murein hydrolase regulator contributes to DNA release and biofilm development in Staphylococcus aureus. Proc Natl Acad Sci USA 2007;104:8113-8118.

32 Ebmeyer J, Furukawa M, Pak K, Ebmeyer U, Sudhoff H, Broide D, Ryan AF, Wasserman S: Role of mast cells in otitis media. J Allergy Clin Immunol 2005;116:1129-1135.

33 Abdullah B, Hassan S, Sidek D, Jaafar H: Adenoid mast cells and their role in the pathogenesis of otitis media with effusion. J Laryngol Otol 2006;120:556-560.

>34 Vogel L, van Alphen L, Geluk F, Jansen H, Dankert J: Opsono-phagocytosis of nonencapsulated Haemophilus influenzae. Adv Exp Med Biol 1995;371A:695-698.

35 Vogel L, van Alphen L, Geluk F, Troelstra A, Martin E, Bredius R, Eijk P, Jansen H, Dankert J: Quantitative flow cytometric analysis of opsonophagocytosis and killing of nonencapsulated Haemophilus influenzae by human polymorphonuclear leukocytes. Clin Diagn Lab Immunol 1994;1:394-400.

36 Walker TS, Tomlin KL, Worthen GS, Poch KR, Lieber JG, Saavedra MT, Fessler MB, Malcolm KC, Vasil ML, Nick JA: Enhanced Pseudomonas aeruginosa biofilm development mediated by human neutrophils. Infect Immun 2005;73:3693-3701.
37 Reid SD, Hong W, Dew KE, Winn DR, Pang B, Watt J, Glover DT, Hollingshead SK, Swords WE: Streptococcus pneumoniae forms surface attached biofilm communities within the middle-ear chamber of experimentally infected chinchillas. J Infect Dis 2008, in press.

38 Bakaletz LO, Tallan BM, Hoepf T, DeMaria TF, Birck HG, Lim DJ: Frequency of fimbriation of nontypable Haemophilus influenzae and its ability to adhere to chinchilla and human respiratory epithelium. Infect Immun 1988;56:331-335.

39 Campagnari AA, Gupta MR, Dudas KC, Murphy TF, Apicella MA: Antigenic diversity of lipooligosaccharides of nontypable Haemophilus influenzae. Infect Immun 1987;55:882-887.

40 Lee N-G, Sunshine MG, Engstrom JJ, Gibson BW, Apicella MA: Mutation of the $h \operatorname{trB}$ locus of Haemophilus influenzae nontypable strain 2019 is associated with modifications of lipid A and phosphorylation of the lipo-oligosaccharide. J Biol Chem 1995;270:27151-27159.

41 Nichols WA, Raetz CRH, Clementz T, Smith AL, Hanson JA, Ketterer MR, Sunshine M, Apicella MA: htrB of Haemophilus influen$z a e$ : determination of biochemical activity and effects on virulence and lipooligosaccharide toxicity. J Endotoxin Res 1997;4: $163-172$.

42 Nichols WA, Gibson BW, Melaugh W, Lee NG, Sunshine M, Apicella MA: Identification of the ADP-L-glycero-D-manno-heptose-6-epimerase $(r f a D)$ and heptosyltransferase II ( $r f a F)$ biosynthesis genes from nontypeable Haemophilus influenzae 2019. Infect Immun 1997;65:1377-1386. 